\section{Obtaining health information on the Internet}

Rapid growth of medical information available on the Internet has occurred over the last few years. Medical training allows us to review and critically appraise the information available. It is clear, however, that many of our patients are also attempting to seek health information online without such training. A recent review article by Cline and Haynes was published in the Journal of Health Education and Research. Over a quarter of a million enquiries are made to the National Library of Medicine (NLM) every day. More than one-third of searches are conducted by patients. What is our responsibility as health care professionals to evaluate the standard of information online? The most widespread attempt at applying a code of conduct to online health information was developed by the Health on the Net Foundation (HON). This is a selfgoverning body that promotes eight ethical standards:

- Advice should be provided by qualified health care professionals.

- References should be clear and other sources with updated information given.

- The evidence presented should be balanced.

- Information should be given clearly.

- Sources of funding should be clearly indicated.

- Advertising should be differentiated from other site contents.

- Confidentiality of user data should be maintained.

- Finally, the type of site should be clearly understood: is it supporting or replacing any existing provider-client relationships?

Websites complying with the HON code display the HON logo.
Source: Cline RJW, Haynes KM, Consumer health information seeking on the Internet: the state of the art, J Health Educ Res 2001, 16(6): 671-692

\section{Update: what advice on air travel and thrombosis?}

The consensus meeting of the World Health Organization (WHO) (March 2001) has suggested a probable link between air travel and thrombosis. The incidence is low, however, and mainly involves passengers with additional risk factors (refer to WHO website). A review article by Giangrande has been recently published in the International Journal of Clinical Practice that summarises current evidence.

- Thrombosis is rarely identified after flights of less than 5 hours; usually flights have been 12 hours or more.

- Risks increase with increasing age (over 50 years).

- Symptoms usually develop within 3 days of the flight.

- Impairment to venous circulation is not exclusive to air travel but is also seen with long car and bus journeys.

- It has been estimated that less than $100 \mathrm{ml}$ of water is lost during long-haul flights, but alcohol excess, diarrhoea and vomiting may increase this

- It is not necessary to get up and walk around during flights; simple leg exercises may be sufficient to promote lower limb circulation.

A recent randomised trial conducted by Scurr et al. published in the Lancet identified a $10 \%$ incidence of asymptomatic deep vein thrombosis (DVT) in both men and women (aged 58-68 years) following long flights. The use of below- knee elasticated stockings offered protection against DVT in a similar group of people. Aspirin has been shown in surgical studies to reduce the risk of arterial and venous thrombosis. The WHO consensus meeting advised against the indiscriminate use of pharmacological agents. Advice to women on contraceptive pill use and air travel should be of a similar nature until more randomised controlled trials have been completed. Additional advice on time delays and pill use, vomiting and diarrhoea, emergency contraception, and condoms purchased outside the UK may also be welcomed by our clients.

Sources: Giangrande PLF, Air travel and thombosis, Int J Clin Pract 2001; 55(10): 690-693; Scurr JH, et al., Frequency and prevention of symptomless deep-vein thrombosis in long-haul flights: randomised trial, Lancet, 12 May 2001: 357: 1485-1489

URL: www.who.int/ncd/cvd/dvt.htm

\section{Directory of hormonal contraception}

The International Planned Parenthood Federation (IPPF) launched the Directory of Hormonal Contraceptives on 18 February 2002. It is available online at www.ippf.org. This comprehensive and user-friendly guide to contraceptives available worldwide allows health care professionals to identify pills with the same hormonal content. The database can be more readily updated than can paper versions. The Directory of Hormonal Contraception is free for a 2-month period but thereafter will be available for a $£ 12$ ( $€ 19)$ subscription fee. URL: www.ippf.org 